\title{
Strong Pluralism, Coincident Objects and Haecceitism
}

\author{
Karol Lenart $^{1} \mathbb{D} \cdot$ Artur Szachniewicz $^{1}$
}

Received: 6 June 2019 / Accepted: 17 September 2019 / Published online: 25 September 2019

(C) The Author(s) 2019

\begin{abstract}
According to strong pluralism, objects distinct by virtue of their modal properties can coincide. The most common objection towards such view invokes the so-called Grounding Problem according to which the strong pluralist needs to explain what the grounds are for supposed modal differences between the coincidents. As recognized in the literature, the failure to provide an answer to the Grounding Problem critically undermines the plausibility of strong pluralism. Moreover, there are strong reasons to believe that strong pluralists cannot provide an explanation of the Grounding Problem. In this paper, we argue that strong pluralism can be motivated independently of the successful answer to the Grounding Problem. In order to achieve that aim, we provide a haecceitistic interpretation of strong pluralism according to which strong pluralism should be read as a position committed to the existence of primitive individuals, i.e., the individuals that have their criteria of individuation independently of their qualitative profiles. That said, we do not aim at defending haecceitism. Instead, our aim is rather modest: we want to provide a new way for the strong pluralist to supplement his view to make it more watertight.
\end{abstract}

Keywords Coincident objects · Pluralism · Identity · Individuals · Haecceitism

\section{Introduction}

Intuitively, a material object is individuated by a portion of matter arranged in certain way; thus, where there is a piece of matter arranged so-and-so, there can only be one object present. Therefore the possibility of multiple material objects located in the same region of spacetime runs against our natural intuitions pertaining to synchronic identity conditions for such objects. No wonder that pluralism, the view that accepts the possibility of distinct coincidents, is not universally endorsed. Even

Karol Lenart

karol.lenart@doctoral.uj.edu.pl

Artur Szachniewicz

artur.szachniewicz@gmail.com

1 Department of Philosophy, Jagiellonian University, Kraków, Poland 
among those sympathetic to the idea, there are two camps. The more moderate weak pluralists accept that material objects coincide temporarily. The more radical strong pluralists allow for spatially and temporally conterminous material objects. The opposition to both is monism, which assigns to each region its own material thing. ${ }^{1}$ We're concerned here with the strong pluralism only. The results should however apply mutatis mutandis to the more agreeable variety.

The position of strong pluralism requires significant argumentation to be worthy of serious consideration. Unless some valid theoretical support can be adduced, it may as well be discarded based on the lack of perceptual evidence to its truth. A case for pluralism is usually made based on a scenario (Gibbard 1975) where two pieces of a raw material are set together to make up a sculpture $S$. The pieces stay in this setting for a short while and get pulverized afterwards. A pluralist will insist that by the mere act of putting the two together, a new piece $L$ was created that coincides with $S$. A monist will deny this, instead advancing the idea that, at best, $S=L$. After all, $S$ and $L$ at each moment of their existence share their external circumstances, are made up of the same quarks and leptons, and are subject to the same mechanical interactions. Their identity is considered to follow from their being materially coincident. Here the pluralist faces the music and comes up with a strong metaphysical thesis that not even permanent material coincidence is enough for identity (cf. Noonan 2015). To make the claim comprehensible, a dispositional or modal difference is sought: $S$ is necessarily $S$-shaped, and $L$ is so only accidentally because $L$ is disposed to take a shape of a flat disc. And if so, given the Leibniz Law, they must be distinct object, the coincidence notwithstanding.

Suppose that pluralism is correct and that material coincidence is not enough to ensure modal properties' conformity. It is only natural to ask what grounds the modal property difference, a challenge known as the Grounding Problem (Burke 1992; Zimmermann 1995; Olson 2001; Bennett 2004). The challenge lurking behind the Grounding Problem is that apparently no valid answer can be given, and thus pluralism is at best committed to modal property primitivism (Bennett 2004). For various reasons, this is not a favourable solution (deRosset 2011). Pluralism can only be legitimately accepted if the modal difference is accounted for, and since the explanation is wanting, no legitimate case for pluralism can be made. Therefore, unless the challenge can either be dismissed or positively met, proffering pluralism is as good as question-begging.

It is likely, though, that the Grounding Problem is not as serious as it seems. First, it may even not be a genuine objection at all, for it is argued that any legitimate explanatory demand it poses on the pluralist can be dismissed and that the demands that cannot be shaken off are in fact illegitimate (deRosset 2011). In particular, contrary to what is sometimes suggested (Noonan 2015, p. 1083), the problem does not track an issue about supervenience, or the lack thereof, on general microphysical properties of the general modal, counterfactual, and dispositional macrophysical

\footnotetext{
1 The distinction between monism and pliuralism follows after Fine (2003). Other authors use their own labels to spell it out (e.g., "multi-thinger" versus “one-thinger") (Bennett 2004), and the distinction between weak and strong pluralism follows (Noonan 2015).
} 
properties of material objects (Zimmerman 1995; Olson 2001; Bennett 2004; deRosset 2011). According to hose authors, there are various types of supervenience that are available for the pluralist. What's important though is that supervenience is not an explanatory relation (Kim 1998), but the Grounding Problem is all about explanation of the modal differences between the coincidents. Second, accepting the legitimacy of the explanatory demand posed by the Grounding Problem, there has already been several approaches at explaining it (see Crane 2012; Sutton 2012). The results of this debate are not the primary issue for us, however. Instead, we focus on another objection whose severity for pluralist might eclipse that of the Grounding Problem, be it legitimate or not.

Insofar as the Grounding Problem challenges pluralism by imposing an explanatory demand which pluralism is supposedly unable to meet, it presents a top-down objection to pluralism's explanation of certain extra-theoretical phenomena. A much more radical move is to deny the very phenomena that the theory is postulated to explain, thus denying the motivation for the theory to be formulated. An objection of this sort has recently been suggested by Noonan (2015).

\section{2 'No Modal Difference' Objection to Strong Pluralism}

As the subtitle suggest, the objection is that the allegedly only modally different coincidents in fact show no modal differences either. In order to present the objection, let us restate the scenario in new terms. An uncontroversial claim, properties come together to constitute complex properties or bundles of properties. Let such bundle of properties be called attributive profiles (A-profiles). The parties to the debate of pluralism seem to accept that wherever there is an A-profile instantiated, there is an individual instantiating it. Thus, they should accept the following framework. First, the properties constitutive of A-profiles can be spread out into two distinct sub-profiles: categorical and modal sub-profiles, respectively C-profiles and M-profiles. Accordingly, for every A-profile, $A=C+M$. Furthermore, a property is an M-property if it is a modal, dispositional, or a counterfactual property, whereas C-properties cover both qualitative and non-qualitative properties, excluding identity properties (for more on why this exclusion is needed, see below). M-properties fall into M-profiles, and C-properties fall into C-profiles. Second, it is rather uncontroversial that A-profiles are individuated by whatever properties constitute them. Thus, for two A-profiles $A_{1}=C_{1}+M_{1}$ and $A_{2}=C_{2}+M_{2}, A_{1} \neq A_{2}$, iff either $C_{1} \neq C_{2}$ or $M_{1} \neq M_{2}$. Third, properties allow for multiple instantiation (Mormann 2012, p. 405); that is, for any property, be it complex or simple, it is possible that it has multiple synchronic instances. Fourth, properties get instantiated by individuals (Rettler and Bailey 2017). Finally, individuals get individuated by the A-profiles they instantiate.

Now, we take that instantiation is a relation holding between an individual and a property, set of properties, or a property and a second-order property. Intuitively, we say that the relation holds, when a given individual $x$ is $F$. Speaking more technically, if a proposition $F(x)$ is true, then $x$ instantiates (exemplifies) $F$ (Orilia and Swoyer 2017). Accordingly, we say that an A-profile $A$ is instantiated if there is an individual $\alpha$ that instantiates the properties constitutive of $A$. 
Therefore, we say that distinct profiles $A_{1}$ and $A_{2}$ are co-locally instantiated at a single region of spacetime if there are distinct individuals instantiating the properties constitutive of $A_{1}$ and $A_{2}$ in such a region. Given that lens, pluralism is a position where there are distinct A-profiles instantiated by distinct but spatiotemporally and materially coincident individuals.

The claims that properties get instantiated by individuals and that individuals get individuated by the A-profiles they instantiate have been contested in serious philosophical debates. First, the claim of multiple instantiation presupposes that property is understood as universal, which precludes particularized properties or properties understood as abstract particulars (Morganti 2007), best known as tropes (Williams 1953; Simons 1994). The claim is justified though, since the admission of tropes renders pluralism trivial. The standard theory of tropes postulates that tropes are primitively individuated particulars. Were it the case that an individual was individuated by its tropes, all individuals that were identical qualitatively would necessarily be numerically identical. Thus, pluralism would be trivially false. However, it seems admissible within trope theory that an individual trope $\tau$ simultaneously inhers in two distinct individuals provided that they are coincident (i.e., if pluralism is true). Thus, trope theory may be said to presuppose pluralism, and its introduction to the debate may be question-begging. Note, however, that we do not assume any particular theory of properties to be true, nor do we assume the falsity of trope theory. We merely restate what is the tacit assumption in the debate: that tropes cannot be included in A-profiles of ordinary objects. Second, there is the individuation claim. In accord with the state of the debate, we assume that A-profiles individuate objects, that is, define what it is to be a given $\alpha$. At this stage, however, we do not want to decidedly answer the question whether objects are reducible to A-profiles or not. That opens possibilities that will be investigated later in this paper.

C-profiles contain both qualitative and non-qualitative properties but not identity properties. To explicate a distinction between these two kinds of properties, one could use a linguistic (Adams 1979; Lewis 1986; Cowling 2015) or metaphysical criterion (Kment 2012). According to the former, a qualitative property is a property that can be formulated in a "language sufficiently rich, without the aid of such referential devices as proper names, proper adjectives and verbs (such as $<$ Leibnizian $>$ or $<$ pegasizes $>$ ), indexical expressions and referential uses of definite descriptions" (e.g., being red or having mass) (Adams 1979, p. 7). In turn, a non-qualitative property is a property that can only be formulated in a language directly referring to a particular individual (e.g., being distinct from Napoleon or being taller than the Eiffel Tower). Equivalently, according to the metaphysical criterion, a qualitative property is a property whose instantiation by a given individual does not depend on which individual it is or to which individual it is related to (Kment 2012, p. 574). Identity properties are a special kind of non-qualitative properties that attribute identity to an individual (e.g., being identical with $\alpha$, where $\alpha$ is a particular individual). If C-profiles contained identity properties, then given that the strong pluralism is a claim that there are distinct objects that share their C-profiles, strong pluralism would be trivially false. 
It is intuitive enough that unless it can be shown that two mutually exclusive A-profiles are co-locally instantiated in spacetime, there can be no valid case made for coincidence. The usual Gibbard-style (Gibbard 1975) strategy involves showing that there are two conflicting A-profiles instantiated in a given region of spacetime $R$. The pluralist needs to identify modal sub-profiles to individuate A-profiles to support this view. Therefore, it is crucial that a pluralist shows that there are real cases of distinct M-profiles co-locally instantiated; the examples are ample.

The Gibbard-style narrative introduced above sets up a paradigm case where different M-profiles ensure that the A-profiles they go into are actually conflicting and that the objects that instantiate them are distinct. A pluralist maintains that for some property $F$ (e.g., being of Elvis' shape and its opposite non-F (e.g., not being of Elvis' shape):

(i) There is an $x$ such that it is possible that $F(x)$.

(ii) There is an $x$ such that it is possible that non- $F(x)$.

Apparently, those properties must be satisfied by distinct individuals. Therefore, $\alpha$ and $\beta$ are introduced as supposedly distinct individuals instantiating distinct M-profiles, hence, distinct A-profiles. The result is that only $\alpha$ can be $F$ and that $\beta$ can be non- $F$. Thus, if the A-profiles are instantiated simultaneously in $R$, they are co-locally instantiated.

Much to the pluralist's chagrin, it can be argued that for any such case of allegedly co-locally instantiated, distinct A-profiles, there really is just one profile instantiated. The argument goes that a strong pluralist overlooks that her construal entails neither that it is not possible that non- $F(\alpha)$ nor that it is not possible that $F(\beta)$, which together with previously introduced (i) and (ii) would be the only case when $\alpha$ and $\beta$ could be said to differ in their capacities (i.e., in the idiom of this paper, be M-profiles). Rather, it is possible that $F(\alpha)$ and it is possible that non- $F(\alpha)$, as well as it is both possible that non- $F(\beta)$ and it is possible that $F(\beta){ }^{2}$ Thus, the purportedly conflicting M-profiles are identical and insofar as they are at all instantiated-they needn't be instantiated by distinct individuals. Since the general theoretical framework described in the beginning states that individuals get individuated by the A-profiles they instantiate, the claim that $\alpha$ and $\beta$ are distinct even though they share their A-profiles is nothing short of absurd. Furthermore, since the main motivation behind pluralism is the observation that there appear to be discrepant A-profiles that are collocally instantiated, showing that the profiles are identical removes any motivation to stipulate distinct individuals to instantiate them.

\footnotetext{
2 Aristotle notices this very fact when he states that "if a thing may be, it may also not be" (De Int. 13, 22b 20). Hintikka calls this notion of possibility "contingency" (Hintikka 1960, p. 20). Thus, the line of thought extended here may only work if $F$ is a contingent property. However, assuming that $\mathrm{F}$ is a not a contingent property would beg the question of pluralism. The idea is that if $F$ is not a contingent property of an $x$, then it's an essential property of that $x$; if there are discrepant essential properties instantiated, it follows there are distinct individuals present. But the point at issue is precisely whether there are distinct individuals present, so we cannot start off with essential properties for the sake of question begging.
} 
Given the argument, pluralism is a non-starter since the circumstances it was meant to account for seem nonexistent. This argument, if valid, also does away with the Grounding Problem. There simply are no distinct profiles collocally instantiated to discuss.

\section{One More Case for Strong Pluralism}

It is possible to recreate the motivation for pluralism by starting from a more general philosophical question. Based on how the world appears, there are certain A-profiles instantiated in various regions of spacetime. Furthermore, let us consider spacetime regions $R_{1}$ and $R_{2}$, such that the A-profiles $A_{1}$ and $A_{2}$ are instantiated in $R_{1}$ and $R_{2}$. If there were no individuals in $R_{1}$ and $R_{2}$, then $A_{1}$ and $A_{2}$ would be instantiated without there being nothing that instantiate them. Were there to be only one individual $\alpha$ coinstantiating them both in their distinct regions, $\alpha$ would have to be bilocated in two distinct locations. Thus, for any distinct non-empty regions, there seem to be located two distinct individuals. Given that $\alpha$ satisfies $A_{1}$ and that $\beta$ satisfies $A_{2}$, it may be worthwhile asking if $\alpha$ and $\beta$ couldn't shift their attributive roles and swap their A-profiles. If so, it should be possible for $\alpha$ to instantiate $A_{2}$ and for $\beta$ to instantiate $A_{1}$. This construal allows for the possibility that there are distinct individuals that have all their properties alike, which is precisely the point made by the strong pluralist.

It turns out one needn't appeal to seemingly discrepant A-profiles that somehow get collocally instantiated to ensure pluralism. Not only does that reinstate strong pluralism as a possibility but turns it into a stronger claim, thus reversing what was considered its main weakness into its strength. Let's call this new position ultrastrong pluralism. The name is justified in as much as the view sanctions the possibility that for a given non-contradictory A-profile $A$, there are multiple individuals exemplifying the properties constitutive of $A$ at one region of spacetime. Thus, the view sanctions that $A$ may be multiply locally instantiated. This follows from the fact that the properties an individual instantiates are not constitutive of its (individual) identity.

Ultra-strong pluralism escapes the Grounding Problem rather easily. Note that the Grounding Problem challenges the strong pluralist's claim that there are distinct M-profiles that individuate individuals instantiating the same C-profiles. If so, the challenge goes, why do the coincidents have distinct M-profiles? However, once the above argument's conclusion is in place, and the coincidents share their M-profiles as well as C-profiles, there is no asymmetric distribution of M-properties. Thus, there is no valid explanatory demand as to why the coincidents display distinct M-profiles.

Instead, as we have seen, a new and more fundamental problem arises for the pluralist. If pluralism is to stay on the table, its supporters have to radically accept that individuals are not individuated by A-profiles containing only C-properties and M-properties. Thus enters ultra-strong pluralism.

To stay abreast with that move, a sceptic (viz. monist) may try to generalise the Grounding Problem. The Generalised Grounding Problem is the demand that 
the pluralist accounts for the pattern of distribution of A-profiles over individuals throughout the ontological domain, whether they coincide or not. However, the generalised explanatory demand is not legitimate and not specific to the ultra-strong pluralist. Again, note that the idea behind the challenge presumably is that unless the pluralist accounts for the individuality of individuals in terms of A-profiles, then she cannot allow herself the idea of distinct individuals at all. But this is begging the question against ultra-strong pluralism, which contends that individuals are not individuated by their respective A-profiles. Insofar as this is the case, the objection is illegitimate. Furthermore, the objection is not specific to arguments on pluralism since it could be used to object to any metaphysical position in the debate of material objects or, broadly, individuals demanding that it accounts for property distribution among individuals. The objection is trivially met only by those theories that stipulate individuals are just bundles of properties (Van Cleve 1985) or tropes (Campbell 1990; Simons 1994).

Having introduced the position, let us now elaborate on what it actually takes to endorse ultra-strong pluralism. First, the commitment to the possibility of objects that preserve identity while swapping their A-profiles commits the ultra-strong pluralist to the falsity of the Principle of Identity of Indiscernibles (henceforth, PII).

PII For any individuals $\alpha$ and $\beta$, if $\alpha$ and $\beta$ have all properties the same, then $\alpha=\beta$.

It's been acknowledged that unspecified PII is trivial (Black 1952; Katz 1983; Rodriguez-Pereyra 2006; Dorato and Morganti 2013). No one opposes that two individuals sharing identity properties are identical, and that follows the assumption that they unrestrictedly share all of their properties. Therefore, to bypass the triviality of PII, one has to reformulate it so that it doesn't presuppose identity or distinctness by including identity-involving properties. Hence, we reformulate PII in terms of A-profiles. By doing this, we can rule out the identity-involving properties given that such properties are excluded from the A-profiles themselves. Following the modification, PII could be read as follows:

\section{PII* For any individuals $\alpha$ and $\beta$, if $\alpha$ and $\beta$ share the same A-profile, then $\alpha=\beta$.}

The ultra-strong pluralist denies PII* precisely by claiming that it is possible for a given individual $\alpha$ to swap its A-profile $A_{1}$ with that of a distinct individual $\beta$ 's, $A_{2}$, without it entailing that $\alpha$ ceases to be or becomes $\beta$. To the contrary, $\alpha$ would preserve its identity when paired with $A_{2}$, likewise with $\beta$ paired with $A_{1}{ }^{3}$ In other

\footnotetext{
${ }^{3}$ In an attempt to defend PII one may appeal to the theory of definite descriptions (Russell 1905) as a tool to help to identify individuals (we would like to thank an anonymous referee for this suggestion). A theory of definite descriptions could help individuate objects if true sentences involving description entailed the existence of a unique designatum of the description. Since there are several approaches to description theory addressing them here with due assiduity is impossible. Therefore, we only point out that Russell-style theory doesn't seem to be of help. Note that the truth of 'the cat is on the mat' does not entail that there's only one cat. Here a qualification may be added that it is the context that helps decide what the description refers to i.e 'the cat' is 'the unique cat in a room we are now sitting'. However, in such a case we already have to know that the designatum exists and is unique. Moreover, Straw-
} 
words, the ultra-strong pluralist claims that individuals might undergo so-called haecceitistic possibilities.

One of the most popular variants of such possibilities is the so-called Black' scenario (Black 1952; Adams 1979), where a possible world with a relational space contains nothing but two iron spheres that have alike internal (monadic, qualitative) and relational properties. As a result, these spheres are indiscernible with respect to their properties, though they aren't identical. According to another example of haecceitistic possibility, there is a possible world that is a qualitative duplicate of our world (i.e., it instantiates the same patterns of A-profiles), yet where neither of us, the authors, exist, for this could be a world where our bodies are inhabited by other persons (Russell 2015). And for a final example, it is possible that the actual world is such that it undergoes an infinite cycle of qualitatively identical recurring epochs. Yet, even though each epoch is like the others, each contains distinct individuals (Lewis 1986).

What is common to all of these different scenarios is that qualitative characteristics of individuals are not sufficient to establish identity criteria for individuals. By applying this to ultra-strong pluralism, one receives a view that A-profiles are not sufficient for establishing identity criteria of their possessors since it is possible for a given individual having some particular A-profile $A_{1}$ to change its profile to some distinct one, say $A_{2}$, and preserve its identity.

A critic might claim that haecceitistic possibilities are not genuine possibilities and that some non-trivial versions of PII (like our PII*) are in fact true. There is a wide discussion over conceivability, for example, about whether it is a reliable guide through possibilities (Yablo 1993; Gendler and Hawthorne 2002). More specifically, some investigate the applicability of conceivability arguments to haecceitistic possibilities (Adams 1979; Cowling 2017). Here we don't want to go into an analysis of these issues since we assume that at least some of haecceitistic possibilities are conceivable. However, even if it turned out that none of them are conceivable for us (humans), it would still be possible to provide a metaphysical rather than epistemological motivation for haecceitistic possibilities.

In order to provide such a metaphysical motivation for haecceitistic possibilities and, thus, demonstrate the falsity of PII*, our ultra-strong pluralist might propose a

\section{Footnote 3 (continued)}

son (1950) pointed out that Russell's theory not so much entails as presupposes the unique designata of descriptions.

Modern approach (e.g. Garson 2013) also doesn't seem to help. Garson introduces an operator ! representing the idea that by using 'the' we mean to pick out a unique individual. Thus "the present king of france" takes a form of !xPx. In his theory the formula !xFx is a term which picks out an individual and has to be supplemented with a predicate to form a truth-apt sentence. The axiom that rules the introduction of terms that contain ! states that if $\mathrm{y}$ is a unique object that poses the feature $\mathrm{F}$, then and only then $\mathrm{y}$ is the object possessing F. Schematically: $(*) \forall y(1 F y \leftrightarrow y=! x F x)$. Now, consider a case of two objects $\alpha$ and $\beta$ each having the same A-profile consisting of a single property F. According to (*) $\alpha$ could be referred to as !xFx only if $\alpha$ were the unique object possessing $\mathrm{F}$, but it's not. The same goes for $\beta$. Ultimately, we already have to know that the object exists and is unique before a definite description is applied to it.

Summing up, we maintain that description theory (at least in its two mentioned variants) cannot rescue PII for it cannot provide a non-circular account of individuation of objects. 
metaphysical explanation of why A-profiles are not sufficient for establishing identities of individuals. By metaphysical explanation, we mean a set of claims about the nature of individuals themselves and not claims pertaining what is possible or not. Henceforth, by metaphysical we mean issues related to non-modal accounts of the nature of individuals, while modal refers to issues about representations of possible worlds, or more broadly, about our modal concepts.

Interestingly enough, such metaphysical motivation for a genuine status of haecceitistic possibilities and denial of PII* is independent from an issue of whether or not haecceitistic possibilities are conceivable or accessible from the perspective of our world. In other words, even if the haecceitistic possibilities were not genuine possibilities or were otherwise inconceivable, it would not entail that PII* is true since there may be some metaphysical (rather than epistemological or modal) reasons for denying PII*. That is, PII* could fail to hold not in virtue of what is possible or what can be known about possibilities but rather in virtue of what is the metaphysical nature of individuals.

In addition, the latter point extends beyond the discussion over the falsity of PII*. It seems that even a proponent of PII* is committed to some metaphysical views about the nature of individuals predicated upon under PII*. This is largely because reference to PII* may merely help determine identity criteria for individuals, which already have to be individuated prior to being predicated of identity or lack thereof. For this reason, it might be worthwhile to distinguish the individuation issue pertaining metaphysical nature of individuals - that is, what individuality of individuals consists of-from the identity issue pertaining to the criteria by which identity or distinctness is predicated by any given pair of individuals already individuated (this will be discussed in more detail later in the text).

Clearly, mere appeals to an identity criterion (like PII*) cannot elucidate all the metaphysical problems about individuals, such as whether there's anything to the metaphysical structure of individuals besides properties. However, since PII* implicitly assumes that individuals are individuated solely by their properties, it itself depends on a view that individuals are nothing over and above bundles of properties (this fact is recognized, for example, by Ladyman and Bigaj 2010; Dorato and Morganti 2013; Møller-Nielsen 2016). Therefore, not only are both parties to the debate about the truth status of PII* subject to supplementing their claims with an explicit metaphysical account of the nature of individuals, the arguments against PII* also have direct bearing on the status of the bundle theory of individuals. Thus, the ultrastrong pluralist providing a case against PII* simultaneously argues against the bundle view of individuals. However, if individuals are not mere bundles of properties, it is natural to ask about what else might play the role of their individuators.

It's important to ask that question in the debate of pluralism because it was observed that if strong pluralism and a fortiori ultra-strong pluralism cannot account for the distinctness of coinciding individuals, then the views are simply absurd (Noonan 2015). The argument, as we understand it, is that since (ultra-)strong pluralism admits but cannot account for what may be considered apparent absurdities (e.g., that objects having the same A-profiles are nonetheless distinct), and since any claim that admits absurdities with no account may itself be considered absurd (ultra-)strong pluralism is absurd. 
However, strong pluralism is absurd only if one holds a view that A-profiles are the only individuators of individuals and, thus, that PII* is true. Moreover, even if it were the case that individuals are nothing over and above bundles of properties and that (ultra-)strong pluralism is in fact false, those facts by themselves wouldn't make the opposite view (accepting the truth of PII* and the bundle theory) any less absurd in the above sense. The bundle theory of individuals is definitely not less problematic (from the metaphysical as well as common-sense perspective) than its opposition, according to which individuals are composed not only of properties but have some additional elements, like bare substrata. Despite the fact that bundle theory, at its roots, has some empiricist or positivist motivations, during the process of development of a bundle theory there emerged many problems that cannot be solved through empirically oriented investigations: What does it take for a bundle of properties to constitute individuals? What is the relation that binds properties together into a bundle? Can individuals survive any slight change of their properties inside the bundle? Could individuals, instead of being a bundle of properties, be identified with bundles of tropes? Having to provide answers to these metaphysical questions, a bundle theorist does not seem to have a much easier position to hold than his opponent. As a result, without a convincing positive metaphysical story behind PII* and the bundle theory, both notions are simply unwarranted hypotheses that might merely happen to be true. Thus, critics of strong pluralism deploying such views still have the ample task of explaining the relevant metaphysical issues associated with their critique. The task of providing such a metaphysical story justifying bundle theory and, a fortiori, PII* is probably of equal difficulty to that the proponent of (ultra-)strong pluralism tackles in his defense of non-qualitative individuators. Below we show that the (ultra-)strong pluralist has some tools to explain away the supposed absurdity of his view and to provide competitive metaphysical motivations for his position.

\section{Kinds of Haecceitism}

We argue that the ultra-strong pluralist can explain his metaphysical claims about the individuation of individuals and falsity of PII* by an appeal to some kind of haecceitism. However, what does haecceitism amount to specifically?

Following a rather overlooked distinction proposed by Fine (2005, see also Stalnaker 2012, p. 59) we would like to distinguish between modal and metaphysical variants of haecceitism. ${ }^{4}$

\footnotetext{
4 It seems that such a distinction is implicitly present in a number of accounts of haecceitism, including the works of Kaplan (1975), Adams (1979), Rosenkrantz (1993), Skow (2008) and Cowling (2012).
} 
Modal haecceitism is a theory about the nature of modality, specifically about a nature of representations of possible worlds. ${ }^{5}$ Given that the units of representations of possible worlds are general (qualitative) and singular (non-qualitative) propositions, a proponent of modal haecceitism claims that for any possible world $W$ in which any general proposition $P$ is true about $W$ and in which any singular proposition $Q$ is true about $W$, the truth of $Q$ does not depend on the truth of $P$. In other words, as Lewis has said (1986, pp. 222-223; Stalnaker 2012; Russell 2015), what is true de re about a given possible world $W$ does not depend on what is true qualitatively about $W$. For example, it might be the case that a possible world $W$ could be characterized by exactly the same general propositions as our actual world W@, yet at $W$ it might be true that Plato was the teacher of Socrates, not the other way around. That is, world $W$ could differ from $W @$ with respect to its de re representation despite no difference with respect to qualitative representation.

According to modal anti-haecceitism, there are no genuine singular propositions about possible worlds. A moderate variant of this view states that what is true de re about possible world $W$ supervenes on what is true qualitatively about $W$. A more radical modal anti-haecceitist would claim that there are no de re truths about possible worlds, fundamentally speaking, but every truth about a possible world is purely general and not about any particular individual.

In light of the presented distinction, it seems that ultra-strong pluralism entails some form of modal haecceitism; for any pair of individuals $\alpha$ and $\beta$, it is possible that there are some singular propositions true about $\alpha$ and $\beta$, respectively, in which their truth values do not depend on the truth values of general propositions completely describing $\alpha$ 's and $\beta$ 's respective A-profiles. From this it follows that there might be modal differences between $\alpha$ and $\beta$ that are independent from how $\alpha$ and $\beta$ 's A-profiles are characterised.

However, as we have indicated earlier on, if one investigates modal properties of individuals (e.g., by embracing modal haecceitism), one should supplement his modal claims about how individuals are represented by some substantive, first-order metaphysical claims about nature of individuals as such. Here there are two general options to choose from, metaphysical haecceitism and metaphysical anti-haecceitism.

In contrast to modal haecceitism, a metaphysical haecceitism is a non-modal thesis about the nature of individuals. In the most general sense, metaphysical haecceitism could be understood as a thesis according to which: (i) there are individuals in the world, and (ii) individuals have an irreducible (primitive) and non-qualitative principle of individuation. Let us call it haecceity. ${ }^{6}$ In the current context, haecceity

\footnotetext{
5 The Plantingian variant of haecceitism is an alternative way of characterising modal haecceitism. According to it, there are non-qualitative individual essences, haecceities (e.g., being identical to Socrates), that are necessary entities and surrogates of every actual or possible entity. Moreover, haecceities ground transworld identity claims. For more details on this view see: Plantinga (1970, 1976), Bennett (2006) and Nelson and Zalta (2009). We will partially refer to this view as a model of the metaphysical conception of haecceities.

${ }^{6}$ However, someone might argue that haecceity is, by definition another formal/qualitative ontological component of individuals and has to be included in A-profiles of individuals. Thus we cannot appeal to haecceity as a non-qualitative ontological component of individuals (We would like to thank an anon-
} 
is just a label for a number of different types of primitive non-qualitative individuators of individuals, such as Scotistic haecceities, thisnesses, bare particulars, substances, and primitive numerical difference. Usually (Adams 1979; Park 1990), haecceities are conceived as primitive in a sense that they are non-qualitative elements of individuals that are irreducible to their qualitative elements, or, in our framework, to any elements of A-profiles. ${ }^{7}$ In light of this, it seems that metaphysical haecceitism entails the falsity of a bundle theory of individuation and, a fortiori, the falsity of PII* (it follows from the fact that PII* assumes the truth of the bundle theory). ${ }^{8}$

There is also a moderate variant of metaphysical anti-haecceitism (sometimes called reductionism; see Salmon 1996; Catterson 2008). According to it, haecceities are not primitive but can be analysed (reduced) in terms of the qualitative character of individuals. One of the most popular variants of this view holds that haecceities are identified with the whole qualitative character of individuals (A-profiles in our framework). ${ }^{9}$ The bundle theory of individuals is a good example of such a moderate metaphysical anti-haecceitistic position. ${ }^{10}$ Other variant of moderate metaphysical anti-haecceitism proposes to analyse haecceities in terms of some unique qualitative elements of individuals (some but not all elements of A-profiles), supposedly, in virtue of essential properties. According to this view, for some individual $\alpha$ to have haecceity $\chi$ is to have some unique essential properties that are parts of $\alpha$ 's A-profile and that profile only. A more radical variant of metaphysical anti-haecceitism is a view that reality does not contain individuals or their haecceities at all.

\section{Footnote 6 (continued)}

ymous referee for highlighting such possibility). The objection's viability depends on what is meant by formal or qualitative element of an individual. If by qualitative we mean the same as some formal component of individuals then haecceity (in any known form) turns out to be qualitative. However, this would entail that every type of component of individuals such as bare particulars, prime matter, prime substance, Scotistic haecceities are also qualitative components of individuals. This raises two problems. First, it runs against how these ontological components were historically understood (they all were understood as non-qualitative components of individuals); Second, it trivialises the notion of qualitativeness for every component of an individual turns out to be qualitative and, the very distinction between qualitative and non-qualitative components stops making any sense.

Now, we rely on a notion of qualitative components of individuals according to which qualitative components of individuals are distinct from non-qualitative ones (see Sect. 2). Under such understanding there are some (orthodox) accounts of haecceities that count it as non-qualitative. Moreover, we argue that these accounts are preferable over their qualitative alternatives (see Sect. 5).

Despite that, even if haecceities were qualitative components of objects it is possible that there is some other yet not qualitative individuator. To put it differently, the idea of non-qualitative individuator deserves consideration even if what so far has seemed most plausibly to be its conceptual representant, i.e. the concept of haecceity, were to be dismissed as another qualitative component of objects.

7 It is important to note however that primitiveness does not entail unanalysability. In fact, we will provide different views on the nature of haecceities even though they are meant to be primitive (irreducible) elements of individuals.

${ }^{8}$ However, the reverse does not hold: from the falsity of PII*, one cannot infer that metaphysical haecceitism is true. This is so due to the fact that falsity of PII* might entail that there are not individuals at all and that generalism is true (we will say more about generalism in a moment).

9 For the discussion of the variant of this view see: Leibniz, Adams (1979), Legenhausen (1989), O'Leary-Hawthorne and Cover (1996) and Møller-Nielsen (2016).

${ }^{10}$ For the discussion of this view see: Van Cleve (1985), Simons (1994). Paul (2010) and Barker and Jago (2018). 
Instead, reality has a purely general and qualitative structure that consists of patterns of properties and/or relations, and that's all there is. The most popular name of this view is generalism, or qualitativism. ${ }^{11} \mathrm{We}$ will not elaborate on this view in any detail since acceptance of it entails the falsity of any form of strong pluralism. In fact, it entails that the whole debate over coincident objects is meaningless since, if true, there would be no objects at all.

In the following section we provide four different views on the nature of haecceities, two reductive ones that rely on some variants of moderate metaphysical antihaecceitism, and two relying on extreme metaphysical haecceitism. We investigate which of the presented models best suit the ultra-strong pluralist's needs and which work against them. We ignore extreme metaphysical anti-haecceitism, since eliminating individuals from ontology renders the debate over individuation and coincidence of objects meaningless.

\section{Metaphysics of Individuals and Ultra-Strong Pluralism}

Below we present four models of haecceities understood as metaphysical components of individuals that individuate them. We claim that three of the presented models suffer from some internal issues that are diffucult to overcome. Moreover, even if those issues were somehow adressed or ignored, the discussed models of haecceities would still remain incompatible with the ultra-strong pluralism. However, there is one option that identifies haecceities with bare particulars that seems to fare better. Yet, our intention is not to defend bare particular theory as such. Our aim is rather modest: to show there is at least one well-established and intelligible way through which the ultra-strong pluralist can explain away the supposed absurdity of his view.

\subsection{Haecceities as A-Profiles}

According to the first variant of moderate metaphysical anti-haecceitism, there are individuals in the world, but their individuality (in our framework, haecceity) is analysable in terms of entire A-profiles of individuals. It results in a view that:

(A) To be the haecceity $\chi$ of an individual $\alpha$ is to be identified with $\alpha$ 's A-profile $A \alpha .^{12}$

In other words, the individuality of individuals consists of nothing more than in their A-profiles; that is, individuals are nothing over and above bundles of their properties. This seems to work hand in hand with PII*, according to which it is not possible to

\footnotetext{
11 For the discussion see: Dasgupta (2009), Turner (2016) and Russell (2015, 2016, 2018).

12 We use a popular essentialistic schema "to be $\alpha$ is to be $\beta$ " to express that it lies in the essence of $\alpha$ that being $\alpha$ is explained by being $\beta$. For more details on the nature of such essentialistic identification see: Correia (2013), Linnebo (2014) and Correia and Skiles (2017).
} 
have distinct individuals $\alpha$ and $\beta$ that share the same A-profile. An argument in favour of such a view might go as follows: suppose that individuals $\alpha$ and $\beta$ share an A-profile $A$ and have their respective haecceities $\chi \alpha$ and $\chi \beta$. If haecceities are analysed in terms of the A-profiles of $\alpha$ and $\beta$, and there are no differences in the A-profiles of $\alpha$ and $\beta$, then haecceities $\chi \alpha$ and $\chi \beta$ are identical. Since it was recognized that haecceities determine the individuality of individuals, it can be concluded that $\alpha$ and $\beta$ are one and the same individual. As a result of such analysis, one receives an account of individuation according to which individuals have haecceities that individuate them. However, since those are analysable in terms of A-profiles, any two individuals $\alpha$ and $\beta$ are identical when they share the same A-profile.

We argue that view (A) has some unacceptable or counterintuitive consequences.

Firstly, (A) entails extreme essentialism: if what it takes to be an individual $\alpha$ is to possess some A-profile $A \alpha$, then every property included in $A \alpha$ is essential for being $\alpha$. In other words, any change to the properties constituting $A \alpha$ entails difference in the haecceity of $\alpha$, but if $\alpha$ had a different haecceity than the one it actually has, it would have been an individual distinct from $\alpha, \alpha^{*}$. Therefore, in every possible world in which $\alpha$ exists, it has to have the A-profile it actually has, $A \alpha$. This means that every property of $A \alpha$ is essential for being $\alpha$.

Secondly, (A) affects the analytic character of all knowledge about features of individuals; suppose that $\alpha$ is nothing over and above $A \alpha$ and that one attributes to $\alpha$ (e.g., Socrates) some property $F$ (e.g., being human) by saying that $\alpha$ is $F$. Assume (for simplicity) that to be Socrates is to be $F, G H$, where $F, G, H$ constitute the A-profile of Socrates. The statement " $\alpha$ is $F$ " therefore becomes " $F, G, H$ is $F$." This is a purely analytic statement. However, this claim conflicts with a very strong intuition that at least some knowledge of properties of individuals is synthetic.

Thirdly, (A) entails that PII* is true, yet there are strong reasons (e.g., haecceitistic possibilities) to believe that PII* is false. Thus, if (A) relies on truth PII*, and if PII* is false, (A) losses one of its main motivations.

Fourthly, even if we ignore the foregoing issues, view (A) would be unacceptable for a proponent of ultra-strong pluralism. The main reason is that if we analyse haecceities in terms of A-profiles, and we deny that there are any additional elements of individuals except A-profiles that might individuate them (by assuming bundle theory of individuation), we are unable to explain what it takes for something to have or instantiate an A-profile. Instead, a proponent of the (A) is committed to a rather counterintuitive claim, that it is an A-profile itself that possess or instantiates the A-profile. In contrast, there is a strong and widely held intuition that properties are possessed by individual objects that are something over and above their properties, and that it is not the case that one and the same property instantiates itself (Orilia and Swoyer 2017). However, even if we put aside the controversiality of this view, it is still incompatible with ultrastrong pluralism. Given that individuality of individuals consist of nothing over and above their A-profiles, the ultra-strong pluralist is unable to maintain that any two distinct individuals $\alpha$ and $\beta$ could possess or exemplify the same A-profile. 


\subsection{Haecceities as Essential Properties}

A moderate metaphysical anti-haecceitist might regroup and claim that individual objects have some unique essential properties belonging to their A-profiles. ${ }^{13}$ In light of that one might propose a variant of moderate metaphysical anti-haecceitism:

(B) To be the haecceity $\chi$ of an individual $\alpha$ is to be a set of essential properties that are parts of $\alpha$ 's A-profile $A \alpha$

According to this view, haecceities of individuals are identified with only some, instead of all, elements of A-profiles. This move makes it is possible to avoid the problem of extreme essentialism discussed above. Instead, view (B) entails only a kind of moderate essentialism according to which only some of the elements of $\alpha$ 's A-profile $A \alpha$ are essential for being $\alpha$. For some it might still be a controversial claim, but it is far less controversial than its extreme counterpart.

Still, there are some internal issues with (B) that render it problematic.

Firstly, (B) entails that every individual object has some essential properties. This follows from the fact that if the individuality of individuals consists of having some essential properties, all individuals must have at least some essential properties. However, it seems plausible that there might be an individual object with only contingent properties that nevertheless make that individual and distinct from every other object.

Secondly, (B) entails a controversial relation between essential properties and A-profiles of individuals: if to be haecceity $\chi$ is to be a collection of essential properties included in a given A-profile, then it is possible for any pair of individuals $\alpha$ and $\beta$ to have distinct A-profiles but share the same collection of essential properties. Thus, it is possible for $\alpha$ and $\beta$ to have the same haecceity, that is, to be identical, even though the remaining non-essential properties in their respective A-profiles are different. Suppose that a proponent of (B) introduces a slightly modified version

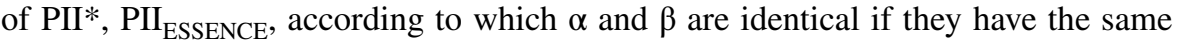
essential properties. But PII $_{\text {ESSENCE }}$ conflicts with PII* since $\alpha$ and $\beta$ would be identical according to $\mathrm{PII}_{\text {ESSENCE}}$, but distinct according to PII*. One way to avoid this consequence would be to argue that given $E$, a collection of essential properties of $\alpha$, it follows that all remaining properties included in $\alpha$ 's A-profile follow from $E$ (and analogically for $\beta$ ). Now, if $\alpha$ and $\beta$ have the same set of essential properties $E$, then it follows that they share all the remaining non-essential properties included in their A-profiles. In such a case, PII $_{\text {ESSENCE }}$ would be consistent with PII*. However, this claim has at least two major issues.

First, it postulates a controversial claim that all elements of A-profiles (except essential properties), in some metaphysical sense, derive from essential properties. However, this seems very mysterious and implausible since it entails that (using Fine's distinction between consequential and constitutive essence, see: Fine 1995) all supposed non-essential properties turn out to be consequentialły essential

$\overline{13}$ A similar view has been recently defended by Barker and Jago (2018). 
properties, meaning they turn out to be essential as a necessary consequence of constitutive essential properties (properties that explain individuality or nature of a given individual) contained in A-profiles. As a result, all properties of individuals would turn out to be essential, and the view (B), contrary the initial motivation for its adoption, would turn out committing to the extreme essentialism. Second, as a consequence of the presented reduction of non-essential properties, the view (B) is in fact identical to the view (A). Because (B) asserts that all non-essential properties follow from essential ones, it turns out that all properties of $\alpha$ and $\beta$ are relevant to the distinctness or identity of $\alpha$ and $\beta$. If so, (B) faces all the problems that (A) faced. Thus, the view (B) would be incompatible with the ultra-strong pluralism for the same reasons as (A).

In response, a proponent of (B) could claim that $\alpha$ has some unshareable essential properties that uniquely characterise $\alpha$ only. However, are there such unshareable essences? Some claim, such as origin essentialists (Kripke 1980; Robertson 1998), that there are such essential properties. The general problem with such essential properties is that, if they are qualitative (e.g., being the fastest man on Earth) or impure (e.g., being faster than Usain Bolt), then it is logically possible that they can be exemplified by distinct individuals at different possible worlds. ${ }^{14}$ This follows (a) from the acceptance of haecceitistic possibilities, which allows for a random qualitative change of individual objects, or (b) from the fact that every qualitative property is a general property and that every impure property is at least partially general. The latter point is supported by the fact that if some property is general or partially general, it can be exemplified by many distinct individuals. If so, a postulate of qualitative or impure essential properties cannot explain the individuality of individuals in a way required by ultra-strong pluralism.

Thirdly, even if a proponent of (B) would somehow overcome the mentioned problems, (B) would still be incompatible with ultra-strong pluralism. If (B) is true, it entails the truth of some non-trivial variant of PII, namely PII $_{\text {ESSENCE: if any pair }}$ of individuals $\alpha$ and $\beta$ have the same essential properties contained in their respective A-profiles, $\alpha$ and $\beta$ have the same haecceities and, thus, are identical. However, acceptance of any non-trivial form of PII conflicts with the basic assumptions of an ultra-strong pluralist, namely that individuals might share their whole A-profiles and nonetheless be distinct objects.

\subsection{Haecceities as Self-Identity Properties}

As we have seen so far, the two previous attempts to analyse haecceities in scope of metaphysical anti-haecceitism are incompatible with intuitions underlying

\footnotetext{
${ }^{14}$ The second example is impure because it is only partially non-qualitative. In this case, a non-qualitative element of a property is a proper name, 'Usain Bolt' that directly refers to a Jamaican former Olympic Champion.
} 
ultra-strong pluralism. ${ }^{15}$ For this reason, we will focus our attention on the analyses based on metaphysical haecceitism. The first variant of this view states:

(C) To be the haecceity $\chi$ of an individual $\alpha$ is to be a property of being $\alpha$ or being identical to $\alpha$, which is part of $\alpha$ 's A-profile $A \alpha$.

Usually properties of being $\alpha$ or being identical to $\alpha$ are understood as non-qualitative individual essences (Plantinga 1970, 1976; Rosenkrantz 1993; Rocca-Royes 2011; Ujvári 2013). Such essences are very often used to provide criteria for transworld identification of individuals or as a proxies of possible individuals, in the case of actualistic theory of possible worlds (Plantinga 1976). In the current context, we would like to make a purely metaphysical usage of non-qualitative individual essences and take them as metaphysical (non-modal) components of individuals that individuate them. ${ }^{16}$ According to the proposed view, there are some unique nonqualitative essential properties of individuals that are unshareable and explain what it takes to be a given individual.

The first problem with this view of haecceities is that the haecceities so understood are dependent on the existence of their bearers. That is, a property $F$ (e.g., being identical with $\alpha$ ) is itself individuated by its relation to $\alpha$. Thus, $\alpha$ has to be individuated independently of F. However, according to (C), it is $F$ that individuates $\alpha$. As a result, to claim that individuality of an individual $\alpha$ consists in having a property $F$ is circular. One cannot (without circularity) explain the individuality of $\alpha$ by an appeal to individual essence $F$ if $F$ is individuated by its relation to $\alpha$ itself.

Secondly, if haecceity is understood as an identity property, the haecceity $\chi$ has to be included in $\alpha$ 's A-profile $A \alpha$ (since A-profiles contain all properties of a given individual). Yet, as we have already shown, A-profiles cannot contain haecceities understood as identity properties since one would be forced to accept a trivialised variant of PII, one which includes identity properties and is trivially true in every case about every object (both for pluralists and monists about coincident objects). In addition, given the acceptance of PII (even in its trivial form), we would be forced to assume bundle theory (since truth of any variant of PII entails truth of some variant

\footnotetext{
15 Someone might claim that there is a further reductive (anti-haecceitist) account of individuality of objects according to which objects are individuated through the external relations they enter, e.g. causal relations. This approach, however, stands in the face of considerable problems. First, modern discussions tend to treat causality as a relation between events, not between objects or events and objects (Schaffer 2016). Second, even if we allow individual objects enter causal relations then, obviously, no sooner can an entity enter a causal relation (or any other suitable external relation) than it is individuated and, so to speak, ready to stand as a relatum in a causal relation; no sooner that it is an individuated object can an entity enter a relation between individuated objects. Thirdly, a proponent of causal view might resist this counter argument by getting rid of the notion of objects at all and claiming that they are either eliminable or reducible to patterns of causal relations (or other suitable relations). This view however is incompatible with the whole idea of coincident objects which presupposes that objects are irreducible ontological category.

16 Adams $(1979,1981)$ explicitly states that non-qualitative individual essences can be understood either as devices for modal identification or as metaphysical components of individuals. He chooses the former reading. We focus on the latter.
} 
of a bundle theory), and despite facing all the internal problems of bundle theory, we would be a priori forced to accept the falsity of ultra-strong pluralism.

Finally, (C) faces a similar problem as (B) and (A): given that the view (C) is correct it would be impossible to explain what it takes for something to possess an A-profile if that thing is identical to its A-profile. Therefore, (C) fails at explaining intuitions lying behind ultra-strong pluralism.

\subsection{Haecceities as Bare Particulars}

Given that all the views on haecceities discussed so far are incompatible with the ultra-strong pluralism, it might be fruitful to abandon the assumption that haecceities are properties (qualitative, impure, or non-qualitative). Most likely it is this very assumption that lies behind the incompatibility of each of the foregoing views with ultra-strong pluralism. As soon as we conceive of haecceities as some kind of property, we are forced to include them in A-profiles. However, as we have indicated, this is the main reason why views (A), (B), and (C) are incompatible with ultrastrong pluralism. In contrast, a view proposed below seems to be free of the two recurring problems of the previous views, namely the problem of circularity and the lacking explanation about objects having properties but just being properties:

(D) To be the haecceity $\chi$ of an individual $\alpha$ is to be a bare particular B of $\alpha$.

According to a standard conception of bare particulars, (Allaire 1963; Bergmann 1967; Moreland 1998), a bare particular is not a property but a further ontological kind of entity that is a constituent of individuals. Therefore, every bare particular metaphysics is an example of a two-categorical ontology (Garcia 2014). According to orthodoxy, bare particulars are propertyless entities that are irreducible to any elements (properties or relations) included in the A-profiles of their bearers. Finally, bare particulars are postulated (rather than discovered) to solve at least three fundamental issues associated with metaphysics of individuals. (Most importantly, these issues need to be explained by ultra-strong pluralist as well. So, if bare particular theory can solve them, it might be a strong reason for ultra-strong pluralist to accept the view D).

Firstly, bare particulars account for haecceitistic possibilities. They explain how it's possible for two objects to share their A-profiles but be distinct. According to bare particular theory, such possibilities are explained by the fact that objects under consideration have different bare particulars but are identical with respect to all their properties. Under the assumption that bare particulars are not properties, and that they do not have any properties (since they are bare), bare particulars provide a noncircular explanation of numerical diversity between any two individuals. ${ }^{17}$

Secondly, bare particulars explain what it takes for an individual to have certain properties or to instantiate a given A-profile. Some individual $\alpha$ instantiates (i.e.,

17 For an orthodox account of bare particulars see: Bergman (1967). 
has) the A-profile $A \alpha$ iff $\alpha$ is constituted by a bare particular $B$ bears $A \alpha$, where bearing is a special way of possessing of properties that is reserved for bare particulars only and is distinguished from the instantiation or the having which is reserved for individuals. For example, Socrates has a property of being human iff Socrates' bare particular $B$ bears a property of being human (Moreland 1998; Wildman 2015).

The difference between these two senses of the possession of properties or whole A-profiles, like $A \alpha$, lies in the fact that while $A \alpha$ is constitutive for individual $\alpha$, it is not constitutive for bare particular $B$ since bare particulars are simple and propertyless. Thus, a connection between A-profiles and bare particulars is less strict than in case of individual objects.

Thirdly, bare particulars explain the unity of properties included in a given A-profile, meaning they explain why properties in a given A-profile are co-exemplified or bundled together. According to this view, such-and-such properties are co-exemplified and constitute an A-profile because there is a bare particular $B$ which exemplifies or bears such-and-such properties.

Obviously, bare particular theory faces some internal problems. First of all, some argue that bare particulars, contrary to the initial assumption, have some properties, like being bare, being simple, or being constituent of one individual at most. Thus, they are not bare at all. This seems to be problematic, for in virtue of what does a given bare particular $B$ have its properties? A plausible answer is that another bare particular $B^{*}$ is constitutive of $B$. This reasoning can be restated for $B^{*}$ and so on, ad infinitum. In response to that, a proponent of bare particulars might insist that bare particulars have no properties at all by accepting a sparse conception of properties according to which not every predicate expresses a genuine property and that the predicates characterising bare particulars do not express genuine properties of bare particulars. One could then maintain that this argument can be reiterated for every other supposed characteristic of bare particulars.

Assuming that this strategy is successful, an opponent of bare particulars might further claim that if bare particulars are genuinely bare, then they are unknowable since one can only know something insofar as one can know its properties. This critique, however, can easily be resisted by recalling that bare particulars are not discovered but postulated. Thus, to complain that we have no cognitive relation to them makes little to no sense and mirrors complaints that a centre of gravity or black matter are cognitively inaccessible to us for they are theoretical postulates.

Someone might also attack a distinction between the different senses of possession of properties proposed: the distinction between instantiating as having properties, on the one hand, and instantiating as bearing properties, on the other. This critique is motivated by the so-called, overpopulation objection to bare particular theory (Bailey 2012; Wildman 2015). According to Bailey, the distinction between bearing and having properties is illusory: if $\alpha$ has $F$ and $B$ bears $F$, then $B$ is $F$ in the same sense that $\alpha$ is $F$. As a result, there are two distinct entities at the same time and place that have the same properties but are not identical. For example, if Socrates is a human, then his bare particular is also a human. So, according to the argument, there are two indiscernible human beings at the same place and time, which seems to be absurd. A proponent of bare particulars could reply to this objection in at least three ways. 
First, how can the argument from overpopulation be a successful complaint against the bare particular theory if one of the main motivations for this theory is to explain the numerical difference between qualitatively indiscernible objects? One cannot argue against bare particular theory by claiming it allows for situations where two objects are qualitatively indiscernible but distinct since, at its bottom, the theory is motivated by the possibility of such scenarios. A proponent of overpopulation, in order to justify his sceptical conclusion, is thus forced to show that individual objects cannot undergo any haecceitistic possibilities. This, however, cannot be established by the overpopulation argument alone, requiring some stronger and more metaphysically loaded arguments (e.g., arguments in favour of non-trivial PII).

Secondly, it might be replied that the sense in which bare particulars bear properties in no way resembles the ordinary having of properties by individuals. For example, if a bare particular of Socrates bears his property of being human, it does not necessitate that the bare particular of Socrates is a human. The bearing of properties refers to an idea that the properties are not constituents of bare particulars but are rather instantiated by them in a primitive, further unanalysable sense of the term (Sider 2006, p. 387-388). That is, individuals have such-and-such properties in virtue of bare particulars bearing or instantiating such-and-such properties.

Thirdly, a proponent of bare particular theory could also abandon the view that bare particulars are mere constituents of individuals and instead identify bare particulars with their host individuals. According to this view, bare particulars are no different from their bearers. For example, if Socrates is a human, then his bare particular is also a human (Sider 2006). Bare particulars just are their possessors (Connolly 2015).

Despite its simplicity and intuitive appeal, this view is incompatible with ultrastrong pluralism. If we claim that bare particulars are identified with their possessors, who are also identified with collections of properties (A-profiles), it entails that bare particulars are identified with collections of properties. This is dictated by the fact that bare particular theory originally postulated only two kinds of ontological constituents of individuals, properties and bare particulars. Now, if one wants to reduce bare particulars, there's nothing left in the ontology to serve as a base for such a reduction except for properties. In response, an alternate identity view could treat individuals as neither identical with bare particulars nor with properties. The problem with this view is that it postulates a primitive category of individual that seems to be on par with that of bare particulars. Primitive bare particulars were meant to be reduced in order not to give rise to the overpopulation problem. However, primitive individuals are susceptible to the same objection. Thus, whenever one attempts to reduce primitive individuals to something else, a regress starts.

As a result, we are left with a view that bare particulars are just identical to collections of properties - that is, to A-profiles themselves. This, however, moves us back to views (A), (B), and (C), and we make no progress at all in motivating ultrastrong pluralism.

Taking these analyses into account, we argue for a variant of bare particular theory that suits the ultra-strong pluralist's needs. This variant adopts rather orthodox view of bare particulars according to which bare particulars are simple, irreducible elements of individuals that bear or instantiate whole A-profiles of their possessors 
and which are irreducible to A-profiles or any elements of them. However, even if the distinction between bearing/instantiating and ordinary having/instantiating cannot be maintained, it is possible to introduce a more modest notion of bare particulars in which bare particulars explain numerical difference between individuals and nothing else. This interpretation is supported by Bergman, who claimed that "bare particulars neither are nor have natures. Any two of them are not intrinsically but only numerically different. That is their bareness. It is impossible for a bare particular to be 'in' more than one ordinary thing ... A bare particular is a mere individuator ... It does nothing else" (Bergman 1967, p. 24-25).

Such a modest notion of bare particulars and their theoretical roles entails that bare particulars cannot explain two issues raised at the beginning of this section: (a) in virtue of what some individual $\alpha$ has its A-profile $A \alpha$ and (b) in virtue of what elements of $A \alpha$ are unified into a single A-profile. If we cannot give an account of bearing or instantiating as distinct relations from ordinary having, then bare particulars are primarily marked with their capability to explain haecceitistic possibilities and to provide an account of the numerical distinctness of individuals. It seems that, pace an opponent of ultra-strong pluralism, such a modest notion of bare particulars does not provide enough resources to answer the Generalised Grounding Problem since it cannot explain in virtue of what a given A-profile $A \alpha$ is exemplified by individual $\alpha$ rather than by individual $\beta$. However, we should not expect bare particular theory to explain away all problems associated with ultra-strong pluralism. Even such a thin bare particular theory is sufficient for our current (modest) purposes since it supports the falsity of PII*, provides a non-circular explanation of individuality of individuals, and explains in virtue of what a given individual has in a given A-profile. The main value of bare particular theory (from the perspective of ultra-strong pluralism) is that it does not reduce being an individual to being a particular A-profile, even explaining that an individual has an A-profile without mentioning A-profiles at all. In other words, if an individual $\alpha$ has the profile it has, then it has it in virtue of $\alpha$ being individuated by bare particular $B$ that primitively instantiates all the properties that constitute the $\alpha$ 's A-profile. Furthermore, $B$ is not a part of $\alpha$ 's A-profile. It is therefore possible to maintain an intuition that it is $\alpha$ that has an A-profile without identifying $\alpha$ with its A-profile.

\section{Conclusions}

We attempted to show that the ultra-strong pluralist has the means to make her position intelligible. We focused on showing that ultra-strong pluralism presumes a quite radical view on the individuation of individuals according to which individuals are not mere bundles of properties but are individuated by some kind of non-qualitative and primitive individuators - broadly speaking, haecceities. We have presented different understandings of haecceities and showed that there is at least one account that suits the ultra-strong pluralist's needs. Despite this, we did not aim to present a definite answer as to what individuates individuals, but we think we have achieved our modest aim to provide a different way for the ultra-strong pluralist 
to try avoiding the supposed absurdity of his position mentioned by Noonan (2015). It seems that if someone might still insist that ultra-strong pluralism faces absurdity, he should also maintain that every variant of metaphysical haecceitism discussed in this paper is absurd also. Such a dismissal of metaphysical haecceitism, however, would be a very controversial claim in need of strong justification. It seems that a proponent of such a radical view might appeal to a bundle theory of individuals or to generalism. However, both theses are far from being uncontroversial.

Acknowledgements The fund was provided by Narodowe Centrum Nauki (Grant No. 2017/25/N/ HS1/01378) and Uniwersytet Jagielloński w Krakowie (Grant No. K/DSC/005588).

Open Access This article is distributed under the terms of the Creative Commons Attribution 4.0 International License (http://creativecommons.org/licenses/by/4.0/), which permits unrestricted use, distribution, and reproduction in any medium, provided you give appropriate credit to the original author(s) and the source, provide a link to the Creative Commons license, and indicate if changes were made.

\section{References}

Adams RM (1979) Primitive thisness and primitive identity. J Philos 76(1):5-26

Adams RM (1981) Actualism and thisness. Synthese 49(1):3-41

Allaire EB (1963) Bare particulars. Philos Stud 14(1):1-8

Bailey AM (2012) No bare particulars. Philos Stud 158(1):31-41

Barker S, Jago M (2018) Material objects and essential bundle theory. Philos Stud 175(12):2969-2986

Bennett K (2004) Spatio-temporal coincidence and the grounding problem. Philos Stud 118(3):339-371

Bennett K (2006) Proxy “actualism”. Philos Stud 129(2):263-294

Bergmann G (1967) Realism: a critique of Brentano and Meinong. University of Wisconsin Press, Madison

Black M (1952) The identity of indiscernibles. Mind 61(242):153-164

Burke MB (1992) Copper statues and pieces of copper: a challenge to the standard account. Analysis 52:12-17

Campbell K (1990) Abstract particulars. Blackwell, Oxford

Catterson TT (2008) Reducing reductionism: on a putative proof for Extreme Haecceitism. Philos Stud 140(2):149-159

Cleve J (1985) Three versions of the bundle theory. Philos Stud 47(1):95-107

Connolly N (2015) Yes: bare particulars! Philos Stud 172(5):1355-1370

Correia F (2013) Metaphysical grounds and essence. In: Hoeltje M, Schnieder B, Steinberg A (eds) Varieties of dependence. Ontological dependence, grounding, supervenience, response-dependence. Philosophia Verlag, München, pp 271-296

Correia F, Skiles A (2017) Grounding, essence, and identity. Philos Phenomenol Res. https://doi. org/10.1111/phpr.12468

Cowling S (2012) Haecceitism for modal realists. Erkenntnis 77(3):399-417

Cowling S (2015) Haecceitism. In: Zalta EN (ed) The Stanford encyclopedia of philosophy. https://plato .stanford.edu/archives/fall2016/entries/haecceitism/. Cited 5 June 2019

Cowling S (2017) Conceivability arguments for haecceitism. Synthese 194(10):4171-4190

Crane JK (2012) Biological-mereological coincidence. Philos Stud 161:309-325

Dasgupta S (2009) Individuals: an essay in revisionary metaphysics. Philos Stud 145(1):35-67

deRosset L (2011) What is the grounding problem? Philos Stud 156:173-197

Dorato M, Morganti M (2013) Grades of individuality. A pluralistic view of identity in quantum mechanics and in the sciences. Philos Stud 163(3):591-610

Fine K (1995) Senses of essence. In: Sinnott-Armstrong W (ed) Modality morality and belief: essays in honor of Ruth Barcan Marcus. Cambridge University Press, Cambridge, pp 53-73

Fine K (2003) The non-identity of a thing and its matter. Mind 112:195-234

Fine K (2005) Modality and tense: philosophical papers. Oxford University Press, Oxford 
Garcia RK (2014) Bare particulars and constituent ontology. Acta Anal 29(2):149-159

Garson JW (2013) Modal logic for philosophers. Cambridge University Press, Cambridge

Gendler TS, Hawthorne J (eds) (2002) Conceivability and possibility. Clarendon Press, Oxford

Gibbard A (1975) Contingent identity. J Philos Logic 4(2):187-221

Hintikka J (1960) Aristotle's different possibilities. inquiry: an interdisciplinary. J Philos 3:18-28

Kaplan D (1975) How to Russell a Frege-Church. J Philos 72:716-729

Katz BD (1983) The identity of indiscernibles revisited. Philos Stud 44(1):37-44

Kim J (1998) Mind in a physical world. MIT Press, Cambridge

Kment B (2012) Haecceitism, chance, and counterfactuals. Philos Rev 121(4):573-609

Kripke SA (1980) Naming and necessity. Harvard University Press, Cambridge

Ladyman J, Bigaj T (2010) The principle of the identity of indiscernibles and quantum mechanics. Philos Sci 77(1):117-136

Legenhausen G (1989) Moderate anti-haecceitism. Res 49(4):625-642

Lewis DK (1986) On the plurality of worlds. Blackwell, Oxford

Linnebo Ø (2014) 'Just is'-statements as generalized identities. Inquiry 57(4):466-482

Møller-Nielsen T (2016) Was Leibniz a Generalist? Studia Leibnitiana 47(1):8-43

Moreland PJ (1998) Theories of Individuation: a reconsideration of Bare Particulars. Pac Philos Q $79(3): 251-263$

Morganti M (2007) Resembling particulars: what nominalism? Metaphysica 8(2):165-178

Mormann T (2012) On the mereological structure of complex states of affairs. Synthese 187:403-418

Nelson M, Zalta EN (2009) Bennett and "proxy actualism”. Philos Stud 142(2):277-292

Noonan H (2015) Against strong pluralism. Philosophia 43(4):1081-1087

O'Leary-Hawthorne J, Cover JA (1996) Haecceitism and anti-haecceitism in Leibniz's philosophy. Noûs 30(1):1-30

Olson ET (2001) Material coincidence and the indiscernibility problem. Philos Q 51:337-355

Orilia F, Swoyer C (2017) Properties. In: Zalta EN (ed) The Stanford encyclopedia of philosophy. https:// plato.stanford.edu/archives/win2017/entries/properties/. Cited 5 June 2019

Park W (1990) Haecceitas and the bare particular. Rev Metaphys 44(2):375-397

Paul LA (2010) Mereological bundle theory. In: Burkhardt H, Seibt J, Imaguire G (eds) The handbook of mereology. Philosophia Verlag, Munich

Plantinga A (1970) World and essence. Philos Rev 79(4):461-492

Plantinga A (1976) Actualism and possible worlds. Theoria 42(1-3):139-160

Rettler B, Bailey M (2017) Object. In: Zalta EN (ed) The Stanford encyclopedia of philosophy. https:// plato.stanford.edu/archives/win2017/entries/object/. Cited 5 June 2019

Robertson T (1998) Possibilities and the arguments for origin essentialism. Mind 107(428):729-750

Roca-Royes S (2011) Essential properties and individual essences. Philos Compass 6(1):65-77

Rodriguez-Pereyra G (2006) How not to trivialise the identity of the indiscernibles. In: Strawson PF, Chakrabarti A (eds) Universals, concepts and qualities: new essays on the meaning of predicates. Ashgate, London, pp 205-224

Rosenkrantz GS (1993) Haecceity: an ontological essay, vol 57. Springer, Berlin

Russell B (1905) On Denoting. Mind 14:479-493

Russell JS (2015) Possible worlds and the objective world. Philos Phenomenol Res 90(2):389-422

Russell JS (2016) Qualitative grounds. Philos Perspect 30(1):309-348

Russell JS (2018) Quality and quantifiers. Aust J Philos 96(3):562-577

Salmon N (1996) Trans-world identification and stipulation. Philos Stud 84(2):203-223

Schaffer J (2016) The metaphysics of causation. In: Zalta EN (ed) The Stanford encyclopedia of philosophy. https://plato.stanford.edu/archives/fall2016/entries/causation-metaphysics/. Cited 5 June 2019

Sider T (2006) Bare Particulars. Philos Perspect 20(1):387-397

Simons P (1994) Particulars in particular clothing: three trope theories of substance. Philos Phenomenol Res 54(3):553-575

Skow B (2008) Haecceitism, anti-haecceitism and possible worlds. Philos Q 58(230):98-107

Stalnaker R (2012) Mere possibilities: metaphysical foundations of modal semantics. Princeton University Press, Princeton

Strawson P (1950) On referring. Mind 59:320-344

Sutton CS (2012) Colocated objects, tally-ho: a solution to the grounding problem. Mind 121:703-730

Turner J (2016) Can we do without fundamental individuals? No. In: Barnes E (ed) Current controversies in metaphysics. Routledge, New York

Ujvári M (2013) Individual essence: gibt es solche? Metaphysica 14(1):17-30 
Wildman N (2015) Load bare particualrs. Philos Stud 172(6):1410-1434

Williams DC (1953) On the elements of being, Parts I and II. Rev Metaphys 7(3-18):171-192

Yablo S (1993) Is conceivability a guide to possibility? Philos Phenomenol Res 53(1):1-42

Zimmerman DW (1995) Theories of masses and problems of constitution. Philos Rev 104(1):53-110

Publisher's Note Springer Nature remains neutral with regard to jurisdictional claims in published maps and institutional affiliations. 